Die Globale Umwelffazilität als zukunftsweisendes Modell

\title{
Umweltpolitik in globalen Netzen
}

\section{Traditionelle (zwischen)staatliche Zusammenarbeit stößt in einer zunehmend globalisierten Welt an die Grenzen ihrer Leistungsfähigkeit. Private Akteure ebenso wie lokale und regionale Gruppen beanspruchen aktive Beteiligung und Einbindung. Die Kooperation aller beteiligten Gruppen ist zu einer Notwendig- keit nachhaltiger internationaler Zusammenarbeit geworden. Am Beispiel des Finanzierungsfonds Global Environmental Facility wird gezeigt, wie internatio- nale Organisationen ihre privilegierte Stellung nutzen können, um Nachhaltig- keit in neven multisektoralen Netzwerken voranzubringen (1).}

D Anzahl von Institutionen, Programmen, Organisationen und Sekretariaten, welche die zwischenstaatliche Zusammenarbeit im Bereich der internationale Umweltpolitik prägen, ist unüberschaubar. Die gegenwärtige Architektur der, global environmental governance" lässt eine Heerschar von Delegierten und Verhandlern ohne Rast um die Welt und die Probleme kreisen. Die Anzahl der tatsächlich erzielten nachhaltigen Erfolge zeigt jedoch eine tiefe Kluft zwischen Anspruch und Wirklichkeit. Der hohe finanzielle und personelle Aufwand steht in keinem Verhältnis zur (ausbleibenden) Verbesserung des Zustandes der globalen Umwelt. Soll der hier beobachtbare Negativtrend umgekehrt werden, dann wird es Zeit, nicht nur die einzelnen Probleme, sondern auch das internationale System, innerhalb welchem Lösungen angestrebt werden, einer kritischen Prüfung zu unterziehen.

\section{Systemschwächen}

Das System der internationalen Zusammenarbeit im Umweltschutz wird bestimmt durch zwischenstaatliche Kooperation in Programmen der Vereinten Nationen (UN), Vertragsstaatenkonferenzen und Sekretariaten, den internationalen Finanzinstitutionen sowie einer Vielzahl weiterer Initiativen, welche in der Regel unter dem Dach einer UN-Organisation oder der Weltbank verwaltet werden. Die verschiedenen Aktivitäten sind in der Regel schlecht koordiniert und fragmentiert. Die Umsetzungs- und Durchführungsdefizite werden durch institutionelle und strukturelle Schwächen verstärkt. Es ist dem System bislang nicht gelungen, sich veränderten globalen Grundvoraussetzungen und neuen Herausforderungen anzupassen. Internationale Organisationen und die traditionell rein zwischenstaatliche Zusammenarbeit stehen heute am Scheideweg. Nur wenn es ihnen gelingt, ihre Rolle neu zu definieren, können nachhaltige Lösungen für die drängenden Probleme unser Welt gefunden werden.

\section{Veränderte Rahmenbedingungen}

Durch die ökonomische und soziale Integration im Rahmen der Globalisierung wird eine steigende Zahl von Problemen, deren Reichweite über die territorialen Grenzen hinausgeht, aus dem rein nationalen Kontext auf die internationale Agenda gehoben. Vor allem die technologische Vernetzung sowie die Liberalisierung und Deregulierung der Märkte führen dazu, dass die weiterhin territorial bestimmten politischen Räume immer häufiger von den grenzüberschreitenden ökonomischen, ökologischen, kommunikativen und sozialen Räumen abweichen.

Während sich staatliche und öffentliche Akteure nur behäbig den Herausforderungen des veränderten Umfeldes stellen, haben sich viele private Akteure schnell global vernetzt. Die Privatwirtschaft als Motor der Globalisierung ist auch ihr erster Nutznießer und agiert und kooperiert seit langem über Ländergrenzen hinweg. Ebenso kooperieren Nicht-Regierungsorganisationen (NROs) heute in transnationalen Netzwerken und reagieren rasch und effektiv auf politische Prozesse und Probleme. Beide Akteursgruppen haben sich auf diese Weise zu beträchtlichen Machtfaktoren entwickelt, die durch Organisation, Struktur und Vernetzung sowie der Möglichkeit, ständig wechselnde Interessenkoalitionen gezielt zu bilden, der staatlichen Zusammenarbeit und den Bürokratien der internationalen Organisationen weit überlegen sind. Da die gegebenen Politik-Strukturen die veränderten Rahmenbedingungen zudem weitestgehend ignorieren, entsteht international auf der Ebene der Partizipation ebenso ein Defizit wie auf der Ebene der operativen Durchsetzung von Entscheidungen.

Betrachtet man die speziellen Probleme der internationalen Governance im Umweltbereich und paart sie mit der Tatsache, dass internationale Organisationen allgemein vor großen Herausforderungen stehen, so scheint ein grundlegender institutioneller Wandel unabdingbar.

\section{- Politiknetzwerke als Alternative}

Netzwerkorientierte Strukturen können an vielen Stellen Lösungen anbieten, an denen müde, langsame Bürokratien nicht in der Lage sind, innovativ und flexibel zu reagieren. Trisektorale Politiknetzwerke zwischen Vertretern von Nationalstaaten, Unternehmen sowie der Zivilgesellschaft eröffnen eine Möglichkeit, den Netzwerken der NROs und der Privatwirtschaft ein politisches Gegenüber anzubieten (2). Globale Politiknetzwerke bringen in dynamischer, nicht-hierarchischer Form die von grenzüberschreitenden Problemen betroffenen Akteure zusammen, um auf der Basis von Interessen- und Wissensdifferenzen in einem ergebnisoffenen Prozess nach tragfähigen Regelungsformen zu suchen. Da dauerhafte Lösungen nur durch die Kooperation aller betroffenen Akteure zustande kommen, sollen nicht nur die betroffenen Sektoren beteiligt werden, sondern vielmehr auch internationale, regionale, nationale und lokale Organisationen bzw. Gruppen. Dabei sollte es zu einer horizontal, vertikal und geographisch angemessenen Verteilung der Partner kommen.

Trisektorale Netzwerke bilden wenig formalisierte Strukturen. In ihnen werden bestimmte Probleme auf die globale Agenda gehoben, Entscheidungen implementiert, Wissen generiert und gesammelt, können aber auch Standards diskutiert, gesetzt und kreative Verhandlungsprozesse geführt werden. Natürlich ranken sich netzwerkähnliche Strukturen zunächst um klar definierte Bereiche und gehen von einzelnen Problemen aus. Sie entstehen in der Regel aus der Situation eines Stillstandes oder einer politischen Lähmung. Finden sich in einer solchen Situation die richtigen Initiatoren in Form einer kleinen Gruppe von engagierten oder betroffenen Schlüsselfiguren, können diese einen politischen Prozess entfachen, der Erfolg versprechendere Lösungen bereithält als die traditionelle, rein zwischenstaatliche Zusammenarbeit.

Netzwerke sind flexible, häufig temporäre Institutionen und in der Lage, Erfahrungen und Wissen 
von Außen zu verarbeiten und auf Probleme schneller zu reagieren als große Bürokratien. Netzwerke sind somit lernfähig. Beispiele für solche netzwerkähnlichen Strukturen sind z.B. die World Commission on Dams oder die Consultative Group on International Agricultural Research (CGIAR).

\section{- Internationale Organisationen und Politiknetzwerke}

Internationale Organisationen können durch die Verlagerung von zentralen, hierarchischen Entscheidungen auf dezentrale Netzwerk-Prozesse eine wichtige Brückenfunktion wahrnehmen (3). Durch ihre internationale Präsenz können sie stimulierend und nicht selten entscheidend zur Bildung von Politiknetzwerken beitragen. Sie verfügen über die Möglichkeiten, die Zusammenarbeit zwischen verschiedenen staatlichen und nicht-staatlichen Akteuren zu fördern, und können Vertreter verschiedener geographischer Regionen mobilisieren und zusammenführen. Weiterhin bieten sie ein geeignetes Forum, Probleme zu definieren und einen Dialog zu entfachen.

Dabei können internationale Organisationen themenorientiert sowohl eine Rolle als Plattform als auch als Mediator wahrnehmen. Sie sind in der Lage, die Bildung von Netzwerken durch das Organisieren von Dialogen zu fördern, sowie Wissen zu sammeln, zu generieren, zu managen und zu verbreiten. Schließlich können sie durch ihre Präsenz und Struktur als Ausführungsorganisationen von Vereinbarungen oder als Wächter über Standards dienen. Letztendlich bietet es sich in einigen Fällen auch an, dass sie aus ihrer eigenen Organisation heraus Netzwerke bilden.

\section{Die Globale Umweltfazilität ...}

Ein Beispiel für eine Institution, die versucht, dezentrale Netzwerkstrukturen von Nicht-Regierungsorganisationen in ihre Entscheidungsprozesse einzubeziehen, und in ihrer eigenen Geschichte immer wieder die Fähigkeit zur Anpassung und Innovation bewiesen hat, ist die sogenannte Globale Umweltfazilität (Global Environment Facility, GEF).

Die GEF wurde im Mai 1991 als Pilotprojekt ins Leben gerufen. Mit einer ersten Finanzausstattung von 860 Millionen US Dollar sollte sie Projekte in den Bereichen Klimaschutz, Artenvielfalt, internationale Gewässer und Schutz der Ozonschicht fördern. Dabei unterstützt die GEF finanziell immer nur den Projektanteil, der einen Beitrag zum globalen Umweltschutz leistet. For- mell überwacht wurde sie in dieser Zeit von der Versammlung ihrer Mitgliedstaaten, wobei die Durchführung der Arbeit bei der Weltbank lag. Diese hatte damit auch weitest gehende Freiheit und Macht über Projektdurchführung, Politik und Strategie der GEF.

Bereits ein ein halb Jahre nach ihrer Gründung wurde die GEF einer tiefgreifenden Reform unterzogen. Eine Restrukturierung war notwendig geworden, nachdem die GEF als Finanzierungsmechanismus für die neuen Konventionen zu Artenvielfalt und Klimaschutz bestimmt wurde. Die Entwicklungsländer (G77) hatten dieser Wahl nur unter der Bedingung einer grundlegenden Reform zugestimmt. Sie fühlten sich in der Pilotphase der GEF nicht ausreichend vertreten, und nahmen sie als von den G7 und der Weltbank dominiert wahr.

Die restrukturierte GEF wird heute von einem institutionell unabhängigen Sekretariat verwaltet. Ihre Entscheidungs- und Verwaltungsstrukturen stellen einen erstaunlichen Kompromiss zwischen den Traditionen der Bretton-Woods-Institutionen Weltbank und Internationaler Währungsfonds sowie der UN dar. Beide Modelle werden von unterschiedlichen Ländergruppen unterstützt und stehen für unterschiedliche Werte, Qualitäten und Schwächen. Die Brücke, welche die GEF zwischen diesen beiden Welten schlägt, steht sinnbildlich für den Kompromiss, der mit der GEF insgesamt auszuhandeln war.

\section{D... als Politiknetzwerk}

Typisch für die GEF ist die außerordentlich starke Einbeziehung von NROs. Während ihrer ganzen Geschichte spielten externe Akteure eine große Rolle. NROs waren an der Entstehung der GEF ebenso beteiligt wie an ihrer Restrukturierung. Sie begleiten die GEF seit zehn Jahren außerordentlich kritisch, wobei nicht zuletzt diese Kritik viele Lernprozesse innerhalb der GEF auslöste. NROs besitzen sowohl eine Stimme bei den Treffen des GEF-Rates als auch ein verbrieftes Recht auf Gehör. Vor allem auf der operativen Ebene ist die Kooperation mit lokalen und internationalen Gruppen unverzichtbar. NROs beraten Ratsmitglieder und beobachten und kommentieren die Projekt-Implementierung, an der sie häufig selbst aktiv beteiligt sind.

Die GEF erwies sich immer wieder als innovationsund anpassungsfähig, da ihre Struktur weniger auf juristische Verbindlichkeit denn auf politischen Kompromiss gegründet ist. Evaluierung und Beurteilung sind heute wichtige Bestandteile.

\section{Modell für Global Governance}

Obwohl die Geschäftstätigkeit der GEF von deutlichen Schwächen gezeichnet ist, die angestrebte Einbeziehung des Privatsektors schleppender als gewünscht verläuft und auch die ungleiche Verteilung von Ressourcen nach wie vor für Asymmetrien in der Machtverteilung sorgt, kann die GEF in verschiedenen Aspekten als Modell für internationale Governance-Strukturen dienen (4):

- Die GEF stellt einen Kompromiss zwischen zwei Strukturmodellen dar, die jeweils von Entwicklungsländern und von entwickelten Ländern favorisiert werden.

- Sie bindet NROs sowohl auf der Entscheidungs- als auch auf der operativen Ebene in ihre Arbeit ein.

- Die GEF basiert auf einem Minimum formaler Vereinbarungen.

- Sie ist eine lernende Institution, in der Lernprozesse gemäß sich verändernder Rahmenbedingungen inszeniert werden.

Überträgt man das Beispiel der GEF auf das Problem der internationalen Governance allgemein, lassen sich einige interessante Feststellungen treffen. Die GEF verabschiedet sich in Teilbereichen von der traditionellen zwischenstaatlichen Zusammenarbeit zu Gunsten der Einbeziehung weiterer Akteure. Ist die GEF als ein traditionell konservativer Finanzmechanismus zu einer solchen Anpassungsfähigkeit in der Lage, müsste dies auch für andere Problem- und Politikfelder gelten. Eine Reform der internationalen Zusammenarbeit im Bereich der Umweltpolitik könnte sich auf ähnliche Netzwerkstrukturen stïtzen.

\section{Ökologischer Strukturwandel} und

\section{Umweltkosten-}

\section{management}

sind die Themen

der Informationsdienste

Ökologisches Wirtschaften

$5 / 01$ und $6 / 01$

Wenn Sie potenzielle Beiträge

haben, wenden Sie sich bitte an die Redaktion. 
Das UN-Umweltprogramm UNEP wäre der natürliche Kandidat, die Arbeit verschiedener Netzwerke zu koordinieren und, soweit möglich, zu managen. UNEP könnte die multisektorale Arbeit durch die Identifikation von Problemfeldern, durch das Zusammenbringen verschiedener Akteure, durch Wissensmanagement und -verbreitung fördern. Um jedoch eine sinnvolle und erfolgreiche Arbeit zu ermöglichen, müsste zunächst eine Strategie entwickelt werden, die einem weiteren ziellosen Aktionismus vorbeugt. Außerdem sollten die verschiedenen internationalen Organisationen nach ihren komparativen Vorteilen eingesetzt werden. Die noch immer vorherrschende Konkurrenz und Verzettelung, die dazu führt, dass personelle und finanzielle Ressourcen unnötig gebunden und verschwendet werden, muss beendet werden und einer fruchtbaren Zusammenarbeit weichen. Zweifellos werden die institutionellen und politischen Widerstände groß sein, doch ohne Vision können die strukturellen Hürden des momentanen Systems der umweltpolitischen Zusammenarbeit nicht überwunden werden.

Auch in diesen Sinne mag die GEF als Vorbild dienen. Sie repräsentiert zwar noch lange nicht den Idealtypus einer netzwerkorientierten $\mathrm{Zu}$ sammenarbeit, hierzu sind unter anderem die institutionellen und politischen Widerstände zu stark. Wie gezeigt stellt sie gleichwohl einen Schritt in die richtige Richtung dar.

\section{Anmerkungen}

(1) Dieser Artikel basiert unter anderem auf der Zusammenarbeit mit Wolfgang Reinicke und seinem Team im Global Public Policy Network Project

(www.globalpublicpolicy.net).

(2) Vgl. zum Folgenden: Reinicke, Wolfgang: Global Public Policy. Governing without Government?, Washington DC 1998 sowie Reinicke, Wolfgang/ Deng, Francis: Critical Choices. IDRC, Ottawa 2000

(3) Vgl. Benner, Thorsten/ Witte, Jan Martin: Brücken im globalen System: Wandel und Zukunft internationaler Organisationen. In: Internationale Politik (im Erscheinen).

(4) Vgl. Streck, Charlotte: The Global Environment Facility - a Role Model for International Governance? In: Global Environmental Politics, Vol. 1, №. 2.

\section{Die Autorin}

Charlotte Streck ist Umweltjuristin bei der Weltbank. Kontakt: The World Bank, MC 6-446; 1818, H-Street, N.W.; Washington, D.C., 20433; USA. Tel. 001-202-4737331, Fax 5221573, E-mail: CStreck@worldbank.org

Internationale Unternehmerorganisationen und die Produktion globaler öffentlicher Güter

\title{
Übersehener Beitrag
}

\author{
Öffentliche Aufgaben werden zunehmend auf private Träger verschoben. Eine \\ solche Privatisierung öffentlicher Politik kann auch im internationalen Rahmen \\ beobachtet werden. Auch wenn Unternehmen als private Governance-Akteure \\ eigene Interessen verfolgen, können sie unter bestimmten Umständen auch \\ einen Beitrag zur Bereitstellung öffentlicher Güter wie Umwelt- und Gesund- \\ heitsschutz leisten. Dies wird am Beispiel Gefahrenminderung bei Farbstoffen \\ illustriert.
}

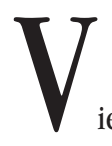
iele globale Regelungs- und Kontrollprobleme sind selbst durch koordiniertes zwischenstaatliches Handeln über internationale Organisationen nicht mehr in den Griff zu bekommen. Der einfache Grund ist, dass es staatlichen Akteuren im Prozess der gesellschaftlichen Differenzierung immer schwerer fällt, die hierfür nötigen Spezialressourcen zu mobilisieren und kollektiven Koordinationsleistungen zu erbringen. Selbst auf nationalstaatlicher Ebene sind staatliche Organisationen in der Formulierung und Implementation öffentlicher Politiken immer mehr auf die Kooperation von privaten Akteuren angewiesen. Derartige Beobachtungen wurden in den vergangenen Jahren unter dem Stichwort Politiknetzwerke diskutiert (1). Eine noch radikalere Antwort auf dieses Problem ist eine Form der Privatisierung öffentlicher Politik, bei der öffentliche Aufgaben insgesamt auf private Träger verschoben werden (2). Solche Prozesse öffentlichen Aufgabentransfers konnten in den vergangenen Jahren auch im internationalen Rahmen beobachtet werden, in dem öffentliche Aufgaben, die bislang von intergouvernementalen Organisationen wahrgenommen wurden, häufig an Nichtregierungsorganisationen (NGOs) delegiert werden.

\section{Neve Governance-Mechanismen}

In den Sozialwissenschaften werden diese institutionellen Arrangements, in denen sowohl öffentliche als auch private Akteure an der Regulierung globaler Entwicklungen mitwirken, unter dem Stichwort Global Governance diskutiert (3). Es wird erkannt, dass viele gesellschaftliche Steuerungsleistungen Abstimmungs- formen jenseits von Markt und Staat erfordern und auf Netzwerke oder andere alternative Governance-Mechanismen verweisen. Insbesondere in Situationen, in denen Marktkontrolle wie staatliche Steuerung unmöglich oder unerwünscht ist, ist globale institutionelle Steuerung auch durch nichtstaatliche Organisationen denkbar. Hierzu werden Organisationen und interorganisatorische Netzwerke im Menschenrechts- und Umweltbereich meist als Beispiele angeführt.

Private Steuerungsbeiträge beschränken sich jedoch nicht auf Organisationen, die unmittelbar an Gemeinwohlzielen orientiert sind, sondern können auch von Privatorganisationen erwartet werden, deren Hauptzweck eher in der Verfolgung von Partikularinteressen liegt. Voraussetzung ist eine Anreizkonstellation, die letztere mit Gemeinwohlinteressen in einer spezifischen Weise verknüpft. Aus einer solchen Perspektive ist es wichtig zu untersuchen, unter welchen Umständen private Organisationen solche Kapazitäten entwickeln können (Ressourcenproblematik), und unter welchen Bedingungen diese hierzu überhaupt das Interesse und den Willen aufbringen (Motivationsstruktur).

In den internationalen Beziehungen wird die Bereitstellung sogenannter öffentlicher Güter durch interstaatliches kollektives Handeln seit Jahrzehnten als interessante theoretische Problematik gesehen (4). Die strukturellen Eigenschaften öffentlicher Güter, Nichtausschlussfähigkeit und Nichtrivalität, implizieren bei einer individualistischen Verhaltenslogik, dass Individuen oder korporative Akteure nur wenig geneigt sind, solche Güter bereitzustellen. Daraus wird meist die Notwendigkeit abgeleitet, für solche Aufgaben öffentliche Organisationen zu errichten, die mit spezi- 
(c) 20I0 Authors; licensee IÖW and oekom verlag. This is an article distributed under the terms of the Creative Commons Attribution Non-Commercial No Derivates License (http://creativecommons.org/licenses/by-nc-nd/3.o/), which permits unrestricted use, distribution, and reproduction in any medium, provided the original work is properly cited. 